\title{
Identification of Fuz7, a Ustilago maydis MEK/MAPKK homolog required for $a$-locus-dependent and -independent steps in the fungal life cycle
}

\author{
Flora Banuett and Ira Herskowitz
}

Department of Biochemistry and Biophysics, School of Medicine, University of California San Francisco, San Francisco, California 94143-0448 USA

\begin{abstract}
Ustilago maydis is a plant pathogenic Basidiomycete fungus that exhibits dimorphism-it has a haploid, yeast-like phase and a dikaryotic, filamentous phase that is pathogenic. Establishment and maintenance of these two forms are controlled by two mating type loci, $a$ and $b$. The $a$ locus is thought to govern fusion of haploid cells to form a dikaryon and is also required for filamentous growth of the dikaryon. It encodes two components of a pheromone response pathway: pheromones and receptors. We report the identification of the U. maydis fuz7 gene, which codes for a putative dual specificity serine/threonine tyrosine kinase of the MAP kinase kinase (MAPKK/MEK) family, by homology with other members of the family. Analysis of mutants deleted for fuz7 shows that it participates in different facets of the life cycle: It is necessary for $a$-locus-dependent processes, such as conjugation tube formation, filament formation, and maintenance of filamentous growth, and for $a$-locus-independent processes, such as tumor induction and teliospore germination. fuz7 is the first $U$. maydis gene distinct from the $b$ locus required for fungal pathogenicity. We propose that fuz7 is involved in at least two pathways, one of which responds to the pheromones coded by the $a$ locus and the other to putative signals from the plant.
\end{abstract}

[Key Words: Signal transduction; fungal dimorphism; pathogenicity; Ustilago maydis; MAP kinase kinase]

Received March 21, 1994; revised version accepted May 3, 1994.

Ustilago maydis, a Basidiomycete fungal pathogen of maize (Zea mays), is dimorphic: The haploid form is unicellular and nonpathogenic; the dikaryotic form is filamentous and pathogenic (for review, see Banuett 1992). The filamentous dikaryon is formed after fusion of two haploid cells that differ at both the $a$ and $b$ matingtype loci. The multiallelic $b$ locus is the major pathogenicity determinant; it encodes a homeo domain protein (for review, see Banuett 1992) that governs filamentous growth as well as pathogenicity. The $a$ locus has two naturally occurring alleles, $a 1$ and $a 2$ (Rowell and DeVay 1954), each of which encodes components of a pheromone response pathway: a pheromone precursor gene and a pheromone receptor gene (Bölker et al. 1992). The putative receptors exhibit amino acid sequence similarity to the Saccharomyces cerevisiae a-factor receptor (Ste3) and to the Schizosaccharomyces pombe Map3 protein (Tanaka et al. 1993), the presumed receptor for $\mathrm{M}$-factor. The presence of these genes at the $a$ locus lends support to the view that the $a$ locus governs cell fusion (Rowell 1955; Trueheart and Herskowitz 1992).

In $S$. cerevisiae, the cell type-specific pheromones and receptors are essential for mating (for review, see Marsh et al. 1991). They are part of a signal transduction path- way that results in morphological changes, cell cycle arrest, cell fusion, and karyogamy. The response pathway includes three serine/threonine protein kinases-Stel1, Ste7, and Fus3/Kss1 - which act sequentially as a kinase cascade (for review, see Neiman 1993; Nishida and Gotoh 1993). A kinase cascade is also involved in the mating-factor response of $S$. pombe. Its members-Byr2, Byrl, and Spk1-exhibit structural and functional similarity to $S$. cerevisiae Ste11, Ste7, and Fus3, respectively (for review, see Neiman 1993; Neiman et al. 1993; Nishida and Gotoh 1993). The fungal Ste11 (Byr2), Ste7 (Byr1), and Fus3 (Spk1) proteins are homologous to the vertebrate kinases MEKK/MAPKKK, MEK/MAPKK, and ERK/MAPK, respectively, which comprise a MAP kinase cascade that is sequentially activated in response to a variety of growth factors (for review, see Crews and Erikson 1993).

S. cerevisiae has two additional MAPK cascades, one involved in osmoregulation (Brewster et al. 1993) and the other in a protein kinase C-mediated signaling pathway (Irie et al. 1993; Lee et al. 1993). The high conservation of these three serine/threonine kinases as a functional module indicates that it is likely to be found in all eukaryotes (Neiman et al. 1993). 
The $U$. maydis a locus is presumed to mediate fusion of haploid cells by activating a pheromone response pathway as in fission and budding yeast. Even after $U$. maydis cells fuse and form a dikaryon, the presence of two different $a$ alleles (together with different $b$ alleles) is still required for filamentous growth (Banuett and Herskowitz 1989|, suggesting that autocrine action of the a-factors is necessary to maintain hyphal growth. It is not known whether the signaling pathway components used in these two phases of the life cycle are the same or whether some components are specific to filamentous (hyphal) growth. Signal transduction might play an additional role in the $U$. maydis life cycle, particularly during fungal differentiation within the tumorous tissue. The $a$ locus does not govern this process (Banuett and Herskowitz 1989|, which has been hypothesized to be induced by plant signals (Banuett 1992).

We report the cloning of $f u z 7$, which codes for a $U$. maydis MEK/MAPKK homolog, by PCR using primers derived from S. cerevisae STE7 and S. pombe byr1. Inactivation of fuz 7 allowed us to infer its role in several facets of the life cycle, some of which are governed by the $a$ locus, and others which are not.

\section{Results}

\section{Identification of fuz 7}

The high degree of identity between STE7 and byr1 was used to design degenerate oligonucleotides that allowed the identification of a Drosophila fragment homologous to STE7 (A. Neiman, pers. comm.). We used the same oligonucleotides (AN9 and AN11; see Materials and methods; Fig. 1) in the polymerase chain reaction (PCR) to identify a STE7 homolog from U. maydis.

Amplification of $U$. maydis total DNA with oligonucleotides AN9 and AN11 yielded a single band of $\sim 350$ bp, whose sequence showed high similarity to STE7 and byr1. The genomic clone containing this gene was isolated from a $U$. maydis cosmid bank using a PCR sibselection strategy (see Materials and methods). This procedure identified cosmid p1 1F23-8, with a $40-\mathrm{kb}$ insert carrying the putative $S T E 7$ homolog.

The sequence of a 1.8-kb $B g I I I$ fragment from cosmid p11F23-8 in plasmid pBB2 (see Materials and methods) revealed an open reading frame (ORF) with coding capacity for 322 amino acid residues, exhibiting a high degree of similarity to byr 1 and $S T E 7$, but lacking coding information for the carboxyl terminus of the kinase domain and beyond. Double-strand sequencing of cosmid p11F23-8 (see Materials and methods) extended the ORF by 113 amino acid residues. Several in-frame stop codons were revealed after these residues, indicating that the $3^{\prime}$ end of the gene had been reached. The complete ORF consists of 435 amino acid residues (Fig. 1); the gene encoding this ORF is designated fuz7.

No consensus splice sites for filamentous fungi (Ballance 1991) were detected in the fuz 7 sequence. The fuz 7 ORF exhibits the highest similarity to mammalian MEK1 (49\% identity), Xenopus MAPKK (47\%), Drosophila Dsorl $(46 \%)$, and S. pombe Byr1 $(46 \%)$ in the catalytic domain (Fig. 2). fuz7 shows $37 \%$ identity to Pbs2, $36 \%$ to Ste 7 , and $35 \%$ to Mkk1 and Mkk2 from S. cerevisiae (Fig. 2) and $35 \%$ with S. pombe Wisl (Warbrick
Figure 1. Nucleotide sequence and deduced amino acid sequence of fuz 7 . The ORF extends from nucleotide +1 to nucleotide 1305 . An asterisk $(*)$ denotes the stop codon. The nucleotides preceding the ATG triplet conform to the fungal consensus, CA/c/a)(a/c)ATGC (Ballance 1991). The $5^{\prime}$-untranslated region is indicated with negative numbers. The arrows show the degenerate oligonucleotides (sense primer AN9 and antisense primer AN11) used to isolate fuz 7 by PCR. The HincII sites at nucleotide positions +88 and +1120 used to create an in vitro deletion of the fuz7 ORF are underlined. (GenBank accession number U07801).

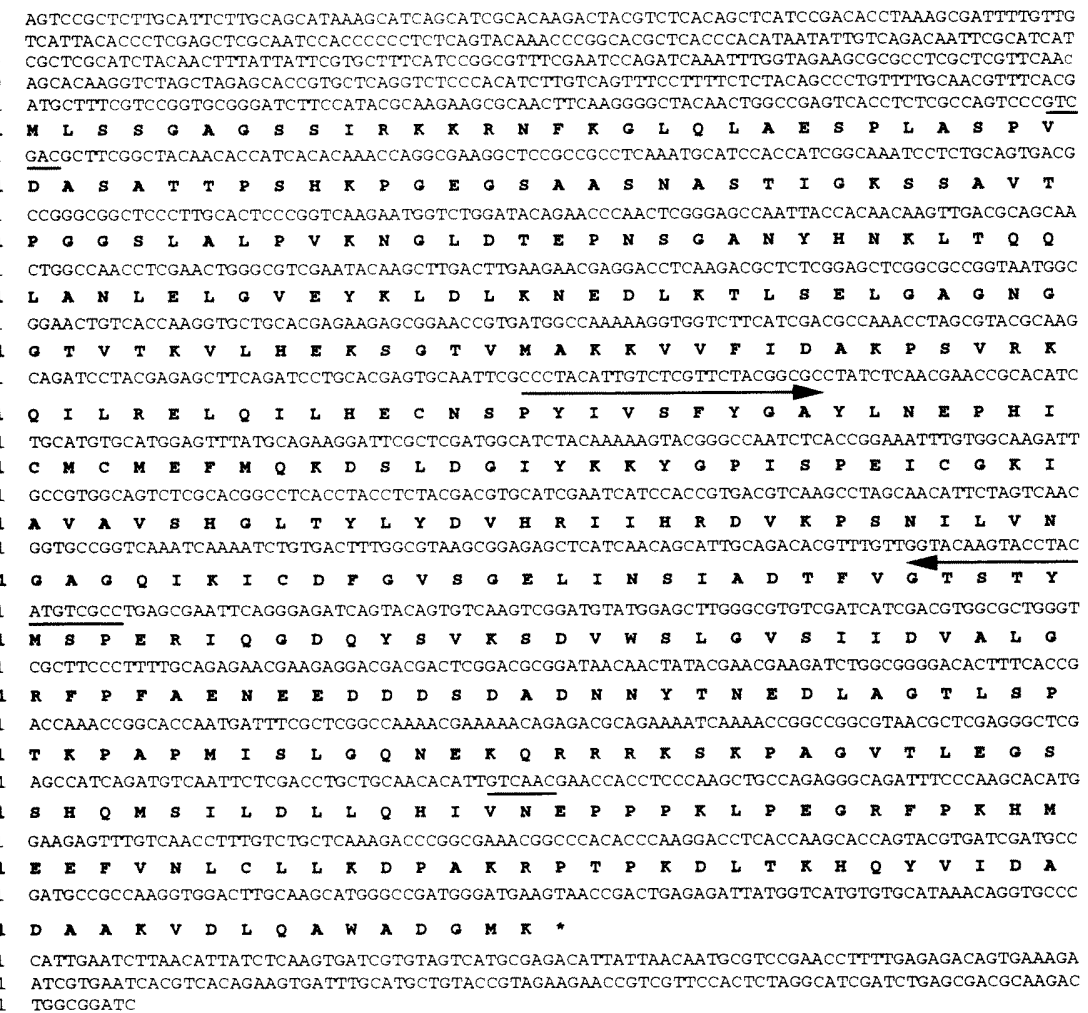




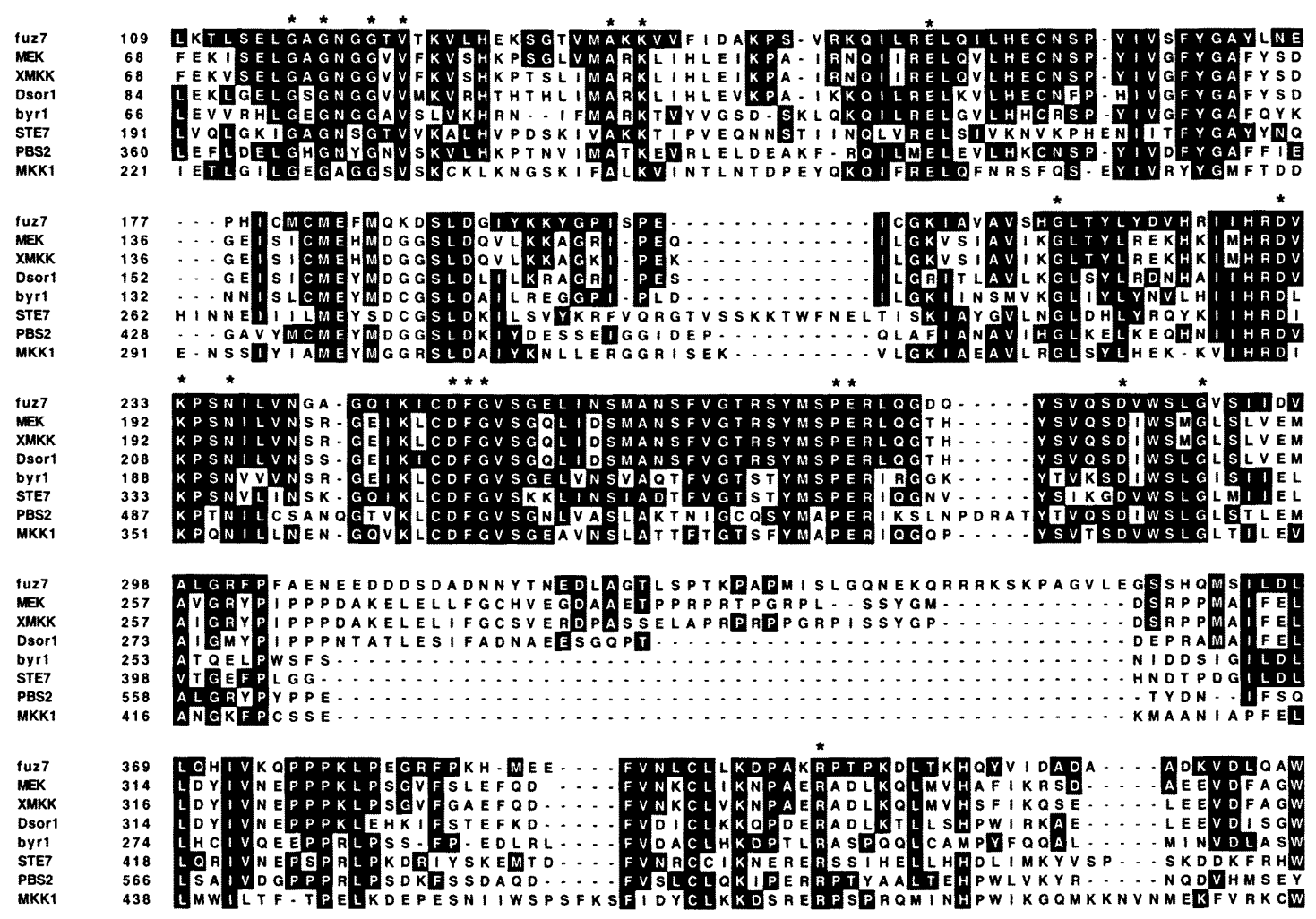

Figure 2. Comparison of the catalytic domain of Fuz7 and other members of the MEK/MAPKK family of serine/threonine/tyrosine protein kinases. The catalytic domain of Fuz7 was compared with that of MEK1 (Crews et al. 1992), Xenopus MAPKK (Kosako et al. 1993), Dsorl (Tsuda et al. 1993), byrl (Nadin-Davis and Nasim 1988), STE7 (Teague et al. 1986), PBS2 (Boguslawski and Polazzi 1987), and MKK1 (Irie et al. 1993). Highlighted amino acids show identities between Fuz7 and any of the other kinases. Asterisks (*| indicate highly conserved amino acids in serine/threonine protein kinases (Hanks et al. 1988).

and Fantes 1991; not shown). All of the highly conserved residues in the catalytic domain of serine/threonine $\mathrm{ki}$ nases (Hanks et al. 1988) are conserved in the fuz7 ORF (Fig. 2). Thus, the fuz7 ORF is structurally similar to the dual specificity kinases (serine/threonine tyrosine $\mathrm{ki}$ nases) of the MEK/STE7 family. Unlike the yeast proteins, the fuz7 ORF contains an insert of 50 amino acids (residues 308-357; see Fig. 2) at the end of subdomain IX (Hanks et al. 1988). Of all the Ste7 family members thus far identified, only the vertebrate protein (MEK1/ MAPKK) contains an insert of similar size to that of Fuz7, though they share little similarity in this region.

\section{Construction of a fuz7 null mutant and analysis of the role of fuz7 in filament formation}

To determine the role of $f u z 7$ in the fungal life cycle, we have constructed a strain deleted for fuz 7 by the one-step gene replacement procedure as described below.

We first constructed a null mutation in fuz 7 in vitro (Fig. 3). This mutation removes the coding information for 364 amino acids, leaving 90 nucleotides at the 5' end of the ORF and 123 nucleotides at its $3^{\prime}$ end (see Figs. 1 and 3), and replaces it with a 3.0-kb PvuII fragment containing the hygromycin B resistance gene /see Materials and methods|. This substitution mutation, denoted
fuz7::hygB, was used to replace the wild-type fuz7 gene of strains $a 1 b 1$ (FB1), a 2 b2 (FB2), a 2 bl met- (FB37-7), and $a 1$ b2 leu $u^{-}$(FB90-8) (Table 1). Hygromycin-resistant transformants were selected (see Materials and methods) and tested for a variety of phenotypes.

Filament formation was tested by cross-streaking the transformants on charcoal agar against appropriate tester strains (see Materials and methods). Filaments are formed only if the two strains that are cross-streaked or cospotted carry different alleles at both $a$ and $b$ (Banuett and Herskowitz 1989|. For all recipients, several transformants exhibited a Fuz ${ }^{-}$phenotype (reduced or no filament formation): $8 / 48$ for FB1, 9/48 for FB2, 20/120 for FB90-8, and 15/110 for FB37-7. Because gene replacement in $U$. maydis is not $100 \%$ efficient (Fotheringham and Holloman 1989|, the Fuz ${ }^{-}$transformants could have resulted either from gene replacement or from integration at nonhomologous loci to generate fuz mutations de novo. To determine which resulted from gene replacement, total DNA from these strains was analyzed by Southern hybridization (see Materials and methods). This analysis indicated that a $1.8 \mathrm{-kb}$ BgIII fragment from the wild-type fuz 7 gene had been replaced by a fragment of slower mobility in $\sim 30 \%$ of the Fuz ${ }^{-}$candidates (at least eight $\mathrm{Fuz}^{-}$candidates from each strain were analyzed; data not shown). Other Fuz ${ }^{-}$strains appear to 


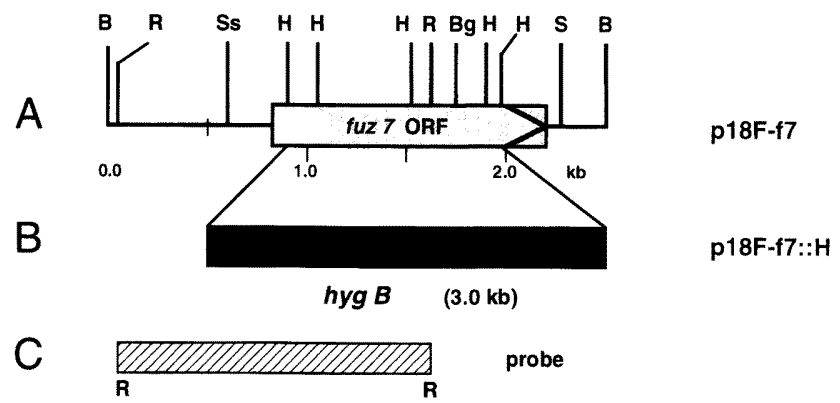

Figure 3. Restriction endonuclease map of the fuz 7 region. $|A|$ The BamHI fragment containing fuz7 in p18F-f7 is a PCR-amplified fragment from cosmid p11F23-8 /see Materials and methods). Restriction endonuclease sites indicated are $\mathrm{BamHI}(\mathrm{B})$; BglII $(\mathrm{Bg}) ; E c o R I(\mathrm{R})$; SspI $(\mathrm{Ss})$; HincII $(\mathrm{H})$; and SphI (S). The BamHI sites correspond in the genomic clone to a BgIII site (left boundary) and a HindIII site (right boundary). (B) p18F-f7::H contains the fuz7::hygB mutation, in which a segment coding for the hygromycin resistance gene (hygB) was used to replace most of the fuz7 ORF (see Materials and methods). The BamHI fragment containing this mutation was used to replace the wildtype gene in strains FB1, FB2, FB37-7, and FB90-8 (Materials and methods). (C) EcoRI fragment used as a probe in Southern hybridization analysis.

carry an insertion of the fragment at nonhomologous loci; other more complex events were also detected. All of the $f u z 7^{-}$strains used in this work carry the fuz7::hygB substitution mutation confirmed by Southern blotting. These mutants exhibit normal growth rates and cell and colony morphology at $25^{\circ} \mathrm{C}, 28^{\circ} \mathrm{C}$, and $32^{\circ} \mathrm{C}$ in rich medium.

The Fuz phenotype of the fuz $7^{-}$strains was retested; representative results are shown in Figure 4. Crossstreaking of wild-type strains $a 2 b 2 \quad \mathrm{fuz}^{+}$and $a 1 \quad b 1$ fuz $7^{+}$yields a vigorous $\mathrm{Fuz}^{+}$reaction after $24 \mathrm{hr}$ [Fig. $4 \mathrm{~A}|\mathrm{c}|)$. In contrast, an $a 2 \mathrm{b2} \mathrm{fuz7^{- }}$ strain cross-streaked against the $a 1 b 1$ fuz $^{+}$strain [Fig. 4A(d)] results in reduced filament formation. A similar result is observed in cross-streaks of an $a 1 \quad b 1 \mathrm{fuz}^{-}$strain with an $a 2 b 2$ fuz $7^{+}$strain [Fig. 4A(e)]. In both cases, the amount of filament formation was greater than produced by crossstreaks of two strains carrying identical $a$ and $b$ alleles [Fig. 4A(f)]. Thus, deletion of fuz 7 results in impaired ability to form filaments, a Fuz ${ }^{-}$phenotype. This phenotype most likely results from impaired ability of the cells to fuse, although it might also result from failure to maintain a stable dikaryon.

The leakiness of the fuz7 null mutation suggests that $U$. maydis has two mating pathways, an efficient fuz7dependent pathway and an inefficient fuz7-independent one. To examine this latter possibility, we cross-streaked an $a 1 b 1 f_{u z 7^{-}}$strain against an $a 2 b 2 f u z 7^{-}$strain [Fig. $4 \mathrm{~A} \mid \mathrm{a}, \mathrm{b})]$. A small amount of filamentous growth is observed after $24 \mathrm{hr}$, although it is reduced in comparison to the $f u z 7^{-} \times f u z 7^{+}$cross-streaks [Fig. 4A(d,e)]; it becomes more evident after $48-72 \mathrm{hr}$ of incubation [Fig. $4 \mathrm{~B}|\mathrm{a}, \mathrm{b}| \mid$. Although this residual filament formation is consistent with the existence of a fuz7-independent pathway, it is also possible that it reflects spontaneous activation of a fuz7-mediated pathway or partial complementation by a fuz 7 relative.

\section{Role of fuz7 in conjugation tube formation}

To study the role of $f u z 7$ and the $a$ locus in early steps of the mating process, we have developed an $a$-locus-dependent assay for cell-cell interaction that measures formation of conjugation tubes (Banuett and Herskowitz 1994). We presume that this morphological response reflects action of the pheromones coded by the $a$ locus.

Table 1. List of strains

\begin{tabular}{|c|c|c|}
\hline Strain & Genotype & Reference/source \\
\hline $\mathrm{FB} 1$ & $a 1 b 1$ & Banuett and Herskowitz (1989) \\
\hline FB2 & $a 2 b 2$ & Banuett and Herskowitz (1989) \\
\hline FB6a & $a 2 b 1$ & Banuett and Herskowitz (1989) \\
\hline FB6b & $a 1 b 2$ & Banuett and Herskowitz (1989) \\
\hline FB37-7 & $a 2 b \mathrm{met}^{-}$ & UV-induced mutant from FB6a \\
\hline FB90-8 & $a 1 b 2 \mathrm{leu}^{-}$ & UV-induced mutant from FB6b \\
\hline FB2-21 & $a 1 b 1$ fuz7::hygB & deletion of $f u z 7$ in $\mathrm{FB} 1$ \\
\hline FB 1-26 & $a 2$ b2 fuz $7::$ hygB & deletion of $f u z 7$ in $\mathrm{FB} 2$ \\
\hline FB7-137 & $a 2 b 1$ met $^{-}$fuz $7:: h y g B$ & deletion of fuz7 in FB37-7 \\
\hline FB8-29 & $a 1$ b2 leu ${ }^{-}$fuz7::hygB & deletion of fuz7 in FB90-8 \\
\hline FBD12 & $a 1 / a 2 b 1 / b 2$ & Banuett and Herskowitz (1989) \\
\hline FBD 12-3 & $a 1 / a 2 b 1 / b 1$ & Banuett and Herskowitz (1989) \\
\hline FBD11-21 & $a 1 / a 2 b 2 / b 2$ & Banuett and Herskowitz (1989) \\
\hline FBD11-7 & $a 1 / a 1 b 1 / b 2$ & Banuett and Herskowitz (1989) \\
\hline FBD $12-17$ & $a 2 / a 2 b 1 / b 2$ & Banuett and Herskowitz (1989) \\
\hline FBD13 & $a 1 / a 2 b 1 / b 2 \mathrm{met}^{-} / \mathrm{met}^{+} 1 \mathrm{eu}-/ 1 e u^{+} \mathrm{fuz}^{+} / \mathrm{fuz} 7^{+}$ & protoplast fusion between FB37-7 and FB90-8 \\
\hline FBD14 & $a 1 / a 2 b 1 / b 2 \mathrm{met}^{-} / \mathrm{met}^{+} 1 \mathrm{eu}-/ 1 e u^{+}$fuz $7:: h y g \mathrm{~B} / \mathrm{fuz} 7^{+}$ & protoplast fusion between FB7-137 and FB90-8 \\
\hline FBD15 & $a 1 / a 2 b 1 / b 2 \mathrm{met}^{-} / \mathrm{met}^{+} 1 \mathrm{eu}^{-} / \mathrm{leu} \mathrm{u}^{+}$fuz $7:: \mathrm{hygB} / \mathrm{fuz}^{+}$ & protoplast fusion between FB8-29 and FB37-7 \\
\hline FBD7-7H & 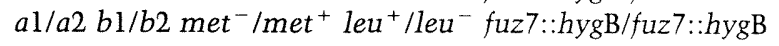 & protoplast fusion between FB7-137 and FB8-29 \\
\hline
\end{tabular}




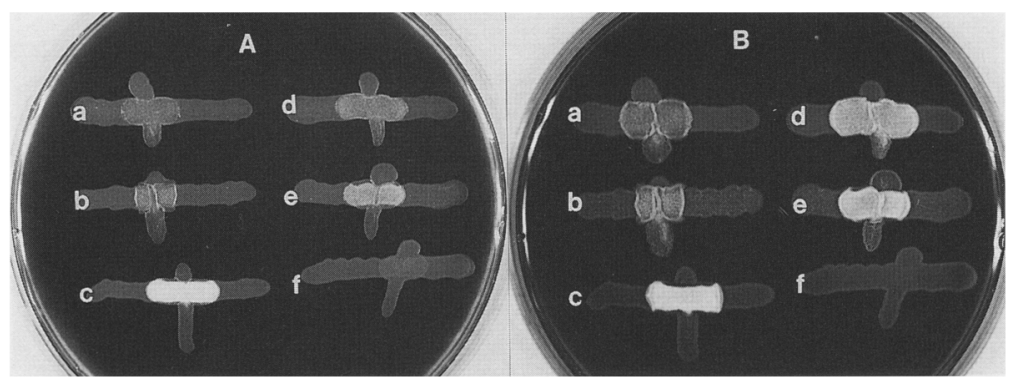

Figure 4. Filament formation by wild-type and fuz7 null mutant strains. Saturated cultures of strains indicated below were cross-streaked on charcoal agar and incubated at room temperature. The white fuzziness that develops at the point of intersection of the cross-streaks is a result of filament formation. Wild-type strains carrying different $a$ and $b$ alleles exhibit a strong reaction after $24 \mathrm{hr}$ (Fuz ${ }^{+}$phenotype; $A c$ ), whereas strains carrying identical $a$ or $b$ alleles do not form filaments, even after long incubation periods $(A f$ and $B f) .(A)$ Reactions after $24 \mathrm{hr}$;

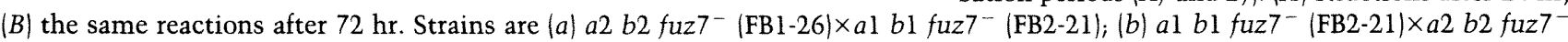

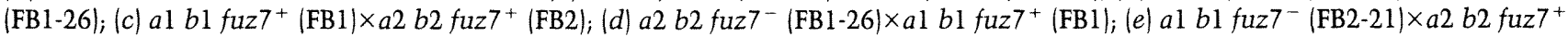
$(\mathrm{FB} 2)$; and $(f) a 1 b 1$ fuz $7^{+}(\mathrm{FB} 1) \times a 1$ bl fuz $7^{+}(\mathrm{FB} 1)$. For unknown reasons, the severity of the fuz $7^{-}$defect appears to be greater in the $a 2$ background than in the $a 1$ background (cf. $d$ and $e$ ).

In this assay, haploid strains that carry different $a$ alleles, regardless of the $b$ allele, form conjugation tubes, with $>90 \%$ of the cells exhibiting a response after $5 \mathrm{hr}$ of coculture under conditions of nutrient limitation (Banuett and Herskowitz 1994). Strains carrying identical $a$ alleles remain arrested as unbudded cells (Banuett and Herskowitz 1994). Thus, the $a$ locus is necessary for conjugation tube formation, which is probably a first step in cell fusion and establishment of the dikaryon.

Coculture of two wild-type strains led to formation of conjugation tubes in $>90 \%$ of the cells in the population (Fig. 5C,D). These conjugation tubes are clearly distinguishable from normal buds of vegetatively growing cells
(Fig. 5A) and do not result simply from starvation (Fig. $5 B)$. In contrast, coculture of a wild-type strain $(a 2 b 2$ fuz $\left.7^{+}\right)$and a fuz7 null mutant $\mid a 1$ b1 fuz $7^{-} \mid$results in a feeble morphological response (Fig. 5E)-only $\sim 5-10 \%$ of the cells begin differentiation. The few conjugation tubes seen at $9 \mathrm{hr}$ of coculture (Fig. 5E) are typical of those observed at $3 \mathrm{hr}$ for wild-type strains (Fig. 5C,D). Even after $24 \mathrm{hr},>95 \%$ of the cells remain arrested, with no signs of morphological differentiation /data not shown). Similar observations were made in coculture of $a 1 b_{1} \mathrm{fuz}^{+}$and $a 2$ b2 fuz $7^{-}$strains (data not shown). These results indicate that neither the fuz ${ }^{+}$nor the fuz strains exhibit a pheromone response. We cannot deter-
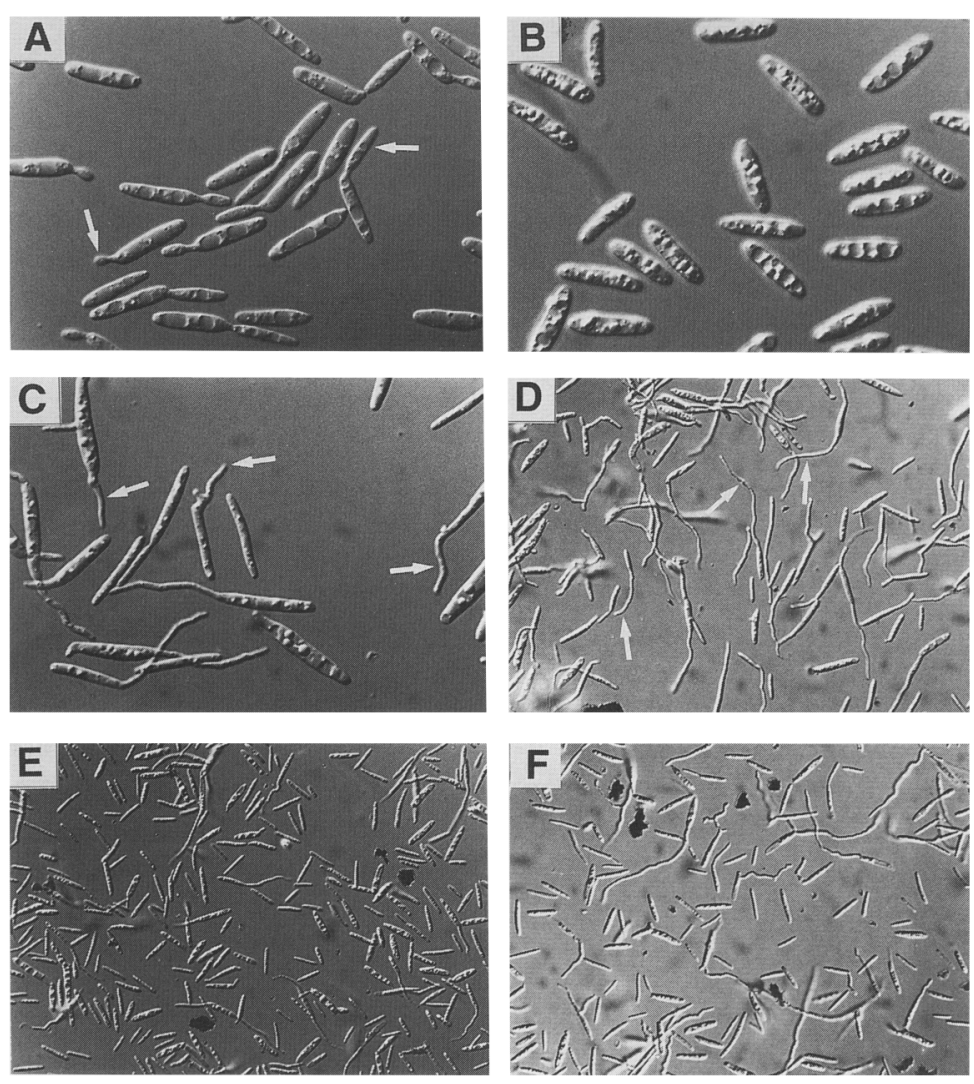

Figure 5. Conjugation tube formation by haploids. $(A)$ An $a$ l bl fuz strain (FB2-21) grown in rich medium exhibits similar growth behavior to that of wild-type haploids. Bud formation is indicated by arrows (magnification, $500 \times) .(B)$ Same strain as in $A$ grown in lownitrogen medium. Cells arrest as unbudded cells $/ \mathrm{mag}$ nification, 500 $\times$ ). (C) Coculture of $a 1 b 1 \mathrm{fuz}^{+}$(FB1) and $a 2 b 2 f u z 7^{+}$(FB2) strains $3 \mathrm{hr}$ after mixing (magnification, $500 \times 1$. The same result is obtained with strains carrying different $a$ alleles and identical $b$ alleles (Banuett and Herskowitz 1994). Arrows point to conjugation tubes. $(D)$ Same as $C$; magnification, $200 \times . \mid E)$ Coculture of $a 1$ b1 fuz7- (FB2-21) and $a 2 b 2 f u z 7^{+}$(FB2) strains $9 \mathrm{hr}$ after mixing (magnification, 200 $\times$ ). $(F)$ Coculture of strains $a 1$ b1 fuz $7^{+}$(FB1), a $2 b 2 \mathrm{fuz}^{+}$(FB2), and $a 1$ b1 fuz $7^{-}$(FB2-21) in a 1:1:1 ratio (magnification, $200 \times 1$. 
mine from this experiment whether failure to form conjugation tubes reflects failure to respond to pheromones, failure to produce pheromones, or both.

To assess the behavior of a fuz $7^{-}$strain under conditions in which pheromones are known to be present, we carried out experiments in which three strains, $a$ l $b 1$ fuz $7^{+}, a 2 b 2$ fuz $7^{+}$, and $a 1 b 1$ fuz $7^{-}$, are cultured together in a 1:1:1 ratio. Approximately two-thirds of the cells exhibited a strong response (Fig. $5 \mathrm{~F}$ ) similar to that observed in coculture of two wild-type strains. A similar experiment in which these three strains were cultured in a ratio of 1:1:2 yielded a morphological response in $\sim 50 \%$ of the cells (data not shown). These results indicate that the fuz $7^{-}$strain is unable to respond to the $a$-locus pheromones. The failure of $a 1 b 1 \mathrm{fuz}^{-}$and $a 2$ $b 2$ fuz $7^{+}$strains to form conjugation tubes when cocultured indicates that the $f u z 7^{-}$strain is defective in pheromone production. High-level production of pheromones may require induction by the pheromone response pathway as in budding yeast (Strazdis and MacKay 1983; Achstetter 19891.

As expected, coculture of two fuz $7^{-}$strains $\mid a \begin{array}{ll}a & b 1\end{array}$ fuz $7^{-}$(FB2-21) and $a 2$ b2 fuz $7^{-}$(FB1-26)] yielded no signs of conjugation tube formation (data not shown). Thus, fuz7 is necessary for the $a$-locus-dependent formation of conjugation tubes.

\section{Role of fuz7 in tumor induction by haploid strains}

To determine the effect of the fuz $7^{-}$mutation on tumor induction, we inoculated 5-day-old maize seedlings with the following strains: (1) $a 1 b 1 f u z 7^{-}(\mathrm{FB} 2-21)$ and $a 2 b 2$ fuz $7^{+}$(FB2) (21 plants inoculated); (2) $a 2$ b2 fuz $7^{-}$(FB126) and $a 1$ b1 fuz $7^{+}$(FB1) (17 plants); (3) $a 2 b 1$ fuz $7^{+}$ (FB37-7) and $a 1$ b2 fuz $7^{-}$(FB8-29) (6 plants); and (4) $a 2$ b1 fuz7- (FB7-137) and $a 1$ b2 fuz $7^{+}$(FB90-8) (6 plants). In all cases, a normal tumor response was observed: Induction of anthocyanin pigmentation, and the time course of tumor development and the size and distribution of the tumors were similar to those observed in fuz $7^{+} \times f u z 7^{+}$inoculations (eight plants inoculated with FB1 and FB2 and six plans inoculated with FB37-7 and FB90-8). The tumors from the $f u z 7^{+} \times f u z 7^{-}$inoculations produced teliospores that germinated normally, yielding segregants in the expected ratios $\left(1 \mathrm{HygB}^{\mathrm{r}} \mathrm{Fuz}^{-}\right.$: $1 \mathrm{HygB}^{\mathrm{s}} \mathrm{Fuz}^{+}$; data not shown). These observations show that tumor induction is normal if one partner is defective in fuz7. Because these infections were performed under optimal conditions with a high inoculum, it is possible that reduced tumor formation might be seen under suboptimal conditions or with lower inocula. As for $\mathrm{fuz} 7^{+}$haploids, plants inoculated with pure cultures of the $f u z 7^{-}$strains do not produce tumors.

In most cases, inoculation of seedlings with haploid strains both deleted for $f u z 7$ resulted in greatly reduced tumor induction: In 11 inoculations with FB2-21 $|a| b 1$ $\left.f u z 7^{-}\right)$and FB1-26 (a2 b2 fuz $\left.7^{-}\right)$and 12 inoculations with FB7-137 (a2 $b 1$ fuz $\left.^{-}\right)$and FB8-29 (al $b 2$ fuz $\left.^{-}\right)$, no tumors were observed at all; in 20 inoculations with FB1-26 and FB2-21 carried out at the same time, small tumors were formed. These tumors produced teliospores in much reduced numbers compared with wild-type or Fuz $7^{+} \times \mathrm{Fuz} 7^{-}$inoculations. In another set of inoculations (with FB1-26 and FB2-21), large tumors comparable in size with those from wild-type inoculations were formed in $8 / 11$ plants inoculated. These tumors contained very few teliospores compared with tumors from wild-type inoculations. The differences in tumor size in these fuz $7^{-} \times f u z 7^{-}$inoculations might be the result of variations in growth conditions. These observations show that fuz 7 is necessary for tumor induction by compatible haploids. Furthermore, fuz 7 is necessary for production of a full crop of teliospores.

\section{Role of fuz7 in maintenance of filamentous growth}

We have shown previously that different $a$ alleles /together with different $b$ alleles) are necessary for maintenance of filamentous growth. These studies (Banuett and Herskowitz 1989| employed diploid strains, which allow examination of postfusion events regardless of any requirements of $a$ or $b$ for establishing a dikaryon. $a 1 / a 2$ $b 1 / b 2$ diploid strains exhibit filamentous growth on charcoal medium, whereas diploids heterozygous only at $a$ or only at $b$ do not.

To determine the role of $f u z 7$ in maintenance of filamentous growth, we constructed a set of isogenic diploid strains heterozygous at both $a$ and $b$ but differing at fuz 7 [fuz $7^{+} /$fuz $7^{+} \mid$FBD13), fuz $7^{-} /$fuz $7^{+} \mid$FBD14 and FBD15), and fuz $7^{-/ f u z 7^{-}}$(FBD7-7H), (Table 1; see Materials and methods)]. Six sets of diploid strains (each consisting of one $f u z 7^{+} / f u z 7^{+}$, one $f u z 7^{+} / f u z 7^{-}$, and one fuz $7^{-} / f u z 7^{-} \mid$were spotted on charcoal agar. All of the $f u z 7^{+} / f u z 7^{+}$and $f u z 7^{-} / f u z 7^{+}$diploids exhibited vigorous mycelial growth after $24 \mathrm{hr}$ [Fuz ${ }^{+}$phenotype; Fig. $6 \mathrm{~A}(\mathrm{a}, \mathrm{b})]$. In contrast, the fuz $7^{-} / f u z 7^{-}$diploids were as deficient in mycelial growth $\left[\mathrm{a} \mathrm{Fuz}{ }^{-}\right.$phenotype; Fig. $6 \mathrm{~A}(\mathrm{c}) \|$ as an $a 1 / a 2 b 1 / b 1 \quad f u z 7^{+} / f u z 7^{+}$control strain [Fig. 6A(d)] at $24 \mathrm{hr}$. After additional incubation for 2-3 days, the fuz $7^{-} / f u z 7^{-}$diploids exhibit some mycelial growth and a characteristic spreading of the spot [Fig. $6 \mathrm{~B}(\mathrm{c})]$. These observations demonstrate that fuz7 is necessary for maintenance of filamentous growth.

Because $a 1 / a 2 b 1 / b 2$ fuz $7^{-} / f u z 7^{-}$diploids exhibit some filamentous growth, $U$. maydis may contain a functionally overlapping gene or a fuz7-independent pathway for filamentous growth.

\section{Role of fuz7 in the dimorphic transition of diploids}

We have shown recently that diploids heterozygous for both $a$ and $b$ are capable of a dimorphic transition from yeast-like growth to hyphal growth when transferred from rich to low-nitrogen medium (Banuett and Herskowitz 1994). These studies indicate that the dimorphic transition has two components: the first, $a$ dependent; the second, $b$ dependent. The $a$-dependent component is exhibited by $a 1 / a 2 b 1 / b 1$ and $a 1 / a 2 \quad b 2 / b 2$ strains, which form what appear to be conjugation tubes. These appendages are short, sinuous, and branched and do not 


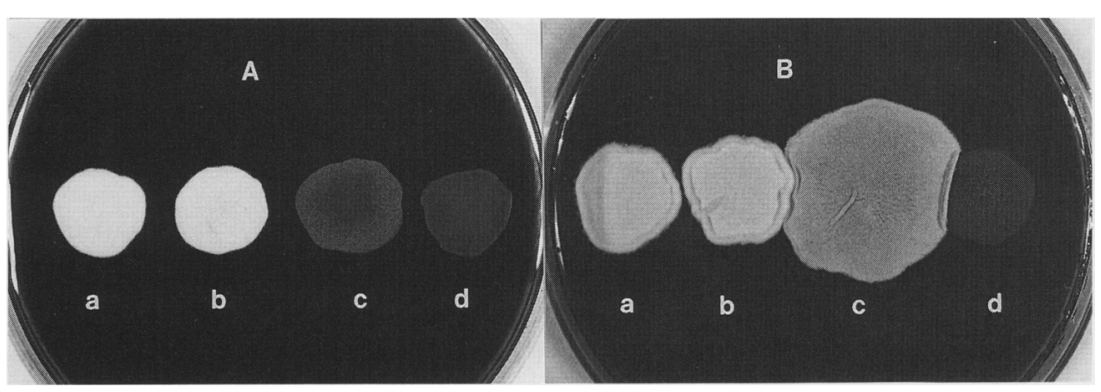

Figure 6. Maintenance of filamentous growth requires fuz7. Fifty microliters of pure cultures of diploids in UMC broth were spotted on charcoal agar and incubated at room temperature: $(A)$ After $24 \mathrm{hr}$; $(B)$ same as $A$ after $72 \mathrm{hr}$. Strains are $(a) a 1 / a 2 b 1 / b 2 f u z 7^{+} /$ fuz $7^{+}(\mathrm{FBD} 13) ;(b) a 1 / a 2 b 1 / b 2$ fuz $7^{+} /$fuz $^{-}$ (FBD15); (c) a1/a2 b1/b2 fuz $7^{-} /$fuz $7^{-}$(FBD7$7 \mathrm{H})$; (d) $a 1 / a 2 \quad b 1 / b 1 \quad f u z 7^{+} / f u z 7^{+}(\mathrm{FB} 12-3)$. The spreading of the spot produced by the fuz $7^{-1} /$ fuz $7^{-}$diploid in $B$ is reproducible; its basis is unknown. develop into hyphae even after prolonged incubation. Because these projections resemble conjugation tubes and their formation is controlled by the $a$ locus, it is likely that their production is under autocrine control by the $a$-locus pheromones. The $b$ component is exhibited by diploid strains heterozygous at both $a$ and $b$. These cells form exuberant, long filaments after shift to low-nitrogen medium (Banuett and Herskowitz 1994).

We have examined the role of fuz7 in this morphological transition by analyzing the behavior of an $a 1 / a 2 \mathrm{bl} /$ b2 fuz $7^{-} /$fuz $7^{-}$diploid (FBD7-7H; Table 1) upon nutritional shift. Its behavior was compared with that of control diploids. As observed earlier (Banuett and Herskowitz 1994), $\mathrm{Fuz}^{+}$al/a2 b1/b2 (FBD12, FBD13, FBD14) strains formed hyphae (Fig. 7A,B) and the Fuz ${ }^{-}$ $a 1 / a 2 b 2 / b 2$ (FBD11-21) strain formed short, conjugation tube-like projections (Fig. $7 \mathrm{C}$ ). In contrast, the fuz $7^{-} / f u z 7^{-}$diploid exhibited no morphological differentiation upon nutritional shift, remaining arrested as unbudded cells without projections even after several days of culture (Fig. 7D). The fuz7 gene is thus necessary for the morphological transition of diploids. These results provide additional evidence that $f u z 7$ is involved in the $a$-dependent autocrine response necessary for maintenance of the filamentous state.

\section{Pathogenicity of fuz $7^{-/ \mathrm{fuz}} 7^{-}$diploids}

Above, we showed that fuz7 is required for normal tumor induction when assayed by coinjection of $a 1 b 1$ $f u z 7^{-}$and $a 2 \quad b 2 \quad f u z 7^{-}$haploid strains. The defect in tumor induction might have resulted solely from inability to form or maintain a dikaryon. To determine whether fuz 7 plays a role beyond formation or maintenance of a dikaryon, we have assayed the ability of $a 1 / a 2$ $b 1 / b 2$ fuz $7^{-/} / f u z 7^{-}$diploids to induce tumors. We therefore inoculated 5-day-old seedlings (see Materials and methods) with a fuz $7^{-} / f u z 7^{-}$diploid (FBD7-7H), as well as with control $f u z 7^{-} / f u z 7^{+}$(FBD14 and FBD15) and fuz $7^{+} / f u z 7^{+}$(FBDl3) diploids. The $f u z 7^{+} / f u z 7^{+}$and fuz $7^{-} / f u z 7^{+}$diploids induced tumor formation in $48 / 53$ plants inoculated. In contrast, the $f u z 7^{-} / f u z 7^{-}$diploids
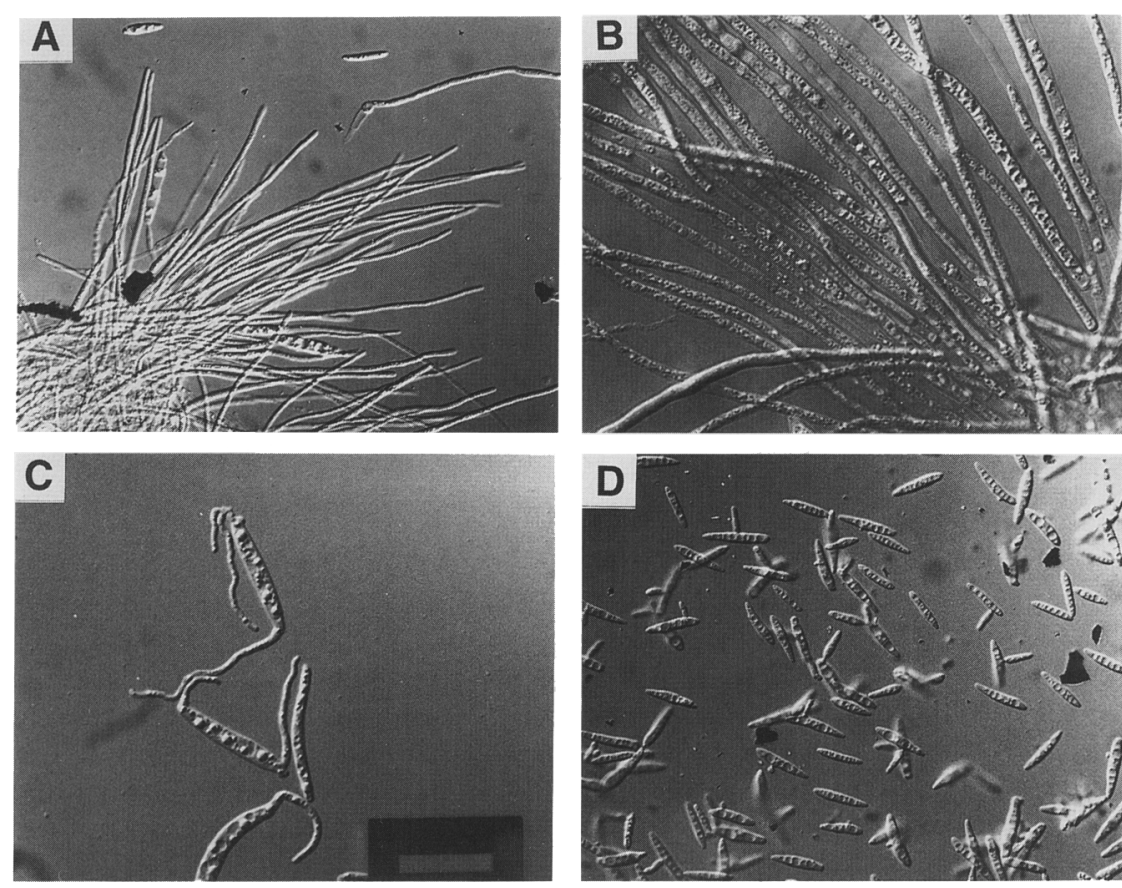

Figure 7. Morphological transition of diploids. Strains were grown in either YEPS or UMC broth and then transferred to low-nitrogen medium. The results shown were recorded $24 \mathrm{hr}$ after shift. (A) Extensive filamentous growth of an $a 1 / a 2 b 1 / b 2 f u z 7^{+} /$ fuz $7^{+}$(FBD13) diploid strain (magnification, $184 \times 1$; it reflects the input of the $a$-dependent and $b$-dependent components of this transition (see text). (B) Filaments (magnification, $460 \times$ ). $(C)$ Appendages exhibited by an $a 1 / a 2 b 2 / b 2 \quad$ fuz $7^{+} / f u z 7^{+}$ (FBD11-21) diploid; they illustrate the $a$-dependent component of the transition. Scale is $20 \mu \mathrm{m}$. Strains homozygous for $a$ and heterozygous for $b$ do not exhibit any morphological transition (data not shown). (D) An $a 1 / a 2 b 1 / b 2$ fuz $7^{-} / f u z 7^{-}$(FBD7-7H) diploid after shift. The growth behavior of fuz $7^{-} / f u z 7^{-}$diploids in rich medium is similar to that of other diploids (Banuett and Herskowitz 1994). 
did not form tumors in $65 / 65$ plants inoculated. We had shown previously that different alleles at $a$ are not necessary for tumor induction once the $b 1 / b 2$ cell is formed: $a 1 / a 1 \quad b 1 / b 2$ (FBD11-7) and $a 2 / a 2 b 1 / b 2$ (FBD12-17) strains induce a normal tumor response (Banuett and Herskowitz 1989). Our new results therefore show that $f u z 7$ is necessary for an $a$-independent process, tumor induction.

Although $a 1 / a 2 b 1 / b 2 f u z 7^{-} / f u z 7^{-}$diploids yield no tumors, we noted earlier that coinfection of $a 1 \mathrm{bl} \mathrm{fuz7^{- }}$ and $a 2 b_{2} \mathrm{fuz}^{-}$haploids results in a low level of tumor induction. This observation suggests that cell fusion (even though reduced in the absence of fuz7) may stimulate tumor induction in some way.

\section{Role of fuz7 in teliospore germination}

Upon germination, the teliospore extrudes a short filament, the promycelium, where meiotic divisions take place (O'Donnell and McLaughlin 1984). To examine the role of fuz7 in this process, the teliospores recovered from the small and large tumors produced by infection with two fuz $7^{-}$haploid strains (see above) were analyzed (see Materials and methods). Of 1700 teliospores from the small tumors, $<1 \%$ germinated; of 200 teliospores from the large tumors, none germinated. In contrast, teliospores from $\mathrm{fuz} 7^{+} \times \mathrm{fuz} 7^{-}$inoculations germinated at a frequency of $85-90 \%$. We had shown previously that diploids homozygous at $a$ and heterozygous at $b$ produce teliospores that germinate normally, indicating that different $a$ alleles are not necessary for teliospore germination (Banuett and Herskowitz 1989). The present results indicate that fuz7 is necessary for promycelium formation, another $a$-locus-independent process.

\section{Discussion}

Development of a multicellular organism not only involves endogenous programming of individual cells but also communication and coordination between cells. Development of a microbial pathogen such as $U$. maydis similarly involves both endogenous programming by fungal regulatory proteins and cell signaling. In this case, signaling not only occurs between fungal cells but also between fungus and host. In addition, the fungus also appears to undergo self-stimulation in an autocrine response.

\section{Fuz7 is a U. maydis MEK/MAPKK homolog}

To learn about the role of signaling in different facets of the life cycle of $U$. maydis, we have used a candidate gene approach to identify a component of such signaling pathways. Recent studies in yeast and in mammalian systems demonstrate the existence of a conserved protein kinase cascade that serves as a functional module for many different signal transduction pathways (for review, see Neiman 1993; Nishida and Gotoh 1993). We therefore chose a member of this cascade, MEK/ MAPKK, and searched for a U. maydis homolog. Using primers designed by similarity to MEK/MAPKK members from yeasts (Ste7 and Byrl), we identified a putative serine/threonine tyrosine protein kinase that we have termed Fuz7. It is likely that this protein is part of a MAPK cascade in $U$. maydis, though confirmation awaits identification of other components in the cascade. As discussed further, below, it is of particular interest to know whether Fuz7 acts on more than one MAP kinase and the nature of the extracellular signals that control its activity.

\section{fuz7 is required for several a-dependent processes}

Because it is possible to construct gene knockouts in $U$. maydis from cloned genes (Fotheringham and Holloman 1989), we were able to inactivate the fuz7 gene and determine its role in different steps of the fungal life cycle. As anticipated, fuz 7 is required for processes requiring the $a$ locus. In particular, fuz 7 is necessary for cells to respond to the $a$-locus pheromones, which we have assayed by induction of conjugation tube formation. In addition, fuz 7 is required for filament formation, maintenance of filamentous growth, and the dimorphic transition of diploids.

Our studies on filament formation suggest that $U$. maydis may execute two modes of cell fusion: an efficient fuz7-dependent mode (requiring different $a$ alleles) and an inefficient fuz7-independent mode. It remains to be determined whether the latter mode reflects unrestricted cell fusion ( $a$-locus independent), as occurs in other Basidiomycetes, notably Coprinus cinereus (Casselton 1978) and Schizophyllum commune (Novotny et al. 1991).

Because different $a$ alleles are necessary to maintain the filamentous state, we have proposed that this form requires autocrine action of the $a$-locus pheromones. The finding that $f u z 7$ is required for maintenance of the filamentous state provides further support for the occurrence of autocrine stimulation. Furthermore, our observations that fuz 7 is necessary for the morphological transition of diploids (from yeast-like growth to filamentous growth) provide additional evidence for an autocrine response involved in filamentous growth. Below, we provide some suggestions for steps that might be controlled by this self-stimulation.

fuz7 is thus required both for interactions between mating partners and for maintaining properties of the product of mating. It is similar in this respect to Ste7, the MEK/MAPKK homolog of S. cerevisiae, which is necessary both for mating between haploid cells (Hartwell 1980 ) and for pseudohyphal development by the a/ $\alpha$ diploid (Liu et al. 1993). In both organisms, the signals for haploid cells are the mating pheromones. Our findings indicate that $U$. maydis dikaryons respond to two signals: the mating pheromones and a putative plant signal (see below). In S. cerevisiae, the signal for the pseudohyphal pathway is clearly not the mating pheromones (Liu et al. 1993), which are not produced in a/ $\alpha$ cells (Duntze et al. 1970|. 
Possible roles of the pheromone response pathway in maintenance of filamentous growth

The requirement of pheromones to maintain filamentous growth of $U$. maydis was unanticipated. It is possible that autocrine action of the pheromones plays a role in coordinating nuclear division, for example, in controlling time of onset of DNA replication. Maintenance of a strict 1:1 ratio of nuclei in hyphal cells necessitates careful control of nuclear division and partitioning of daughter nuclei. It is also possible, at least in planta, that maintenance of dikaryosis in $U$. maydis involves a complex process like that exhibited by $\mathrm{C}$. $\mathrm{ci}$ nereus and S. commune (for review, see Casselton 1978; Novotny et al. 1991), in which a special appendage, the clamp connection, is formed by the tip cell. It is tempting to make an analogy between fusion of the clamp connection with the penultimate hyphal cell and the process of facilitated fusion that occurs in mating between $U$. maydis haploids.

\section{Fuz7 is required for a-independent processes}

Although the $a$ locus governs establishment and maintenance of the dikaryotic state, it does not appear to control pathogenesis and other developmental events that ensue when $U$. maydis infects its host. In particular, different $a$ alleles are not necessary for induction of tumors in the host or for fungal differentiation within the tumors once the $b 1 / b 2$ cell is established. Because the $a$ locus does not govern these processes, it was intriguing to find that the fuz 7 mutants exhibit a severe defect in tumor induction and teliospore production. Furthermore, the few teliospores that are formed are themselves deficient in germination. We interpret the failure of $f u z 7$ mutants to induce tumors to indicate that the Fuz7 protein may play a role in a signaling pathway distinct from that involved in response to the $a$-locus pheromones. In particular, we hypothesize that fuz 7 may be activated in response to signals from the host plant in the process leading to disease development. fuz 7 is the first gene of $U$. maydis aside from the $b$ locus (a regulatory locus) to be directly implicated in pathogenicity.

It is thought that reciprocal signaling between pathogen and host plays an important role in disease development. The most intensively studied cases of such reciprocal signaling are provided by the interaction of the bacterial pathogen Agrobacterium tumefaciens with its hosts in crown gall disease (for review, see Long and Staskawicz 1993), and by the interaction of Rhizobium (a symbiont) with legumes in nodulation (see Dénarié and Cullimore 1993; Long and Staskawicz 1993). Identification of the $U$. maydis fuz7 gene is a first step in unravelling the interactions between this fungus and its host.

Fungal differentiation, a process during which karyogamy occurs and a specialized pigmented cell wall is deposited, results in formation of diploid spores (teliospores) within the tumors. This process is independent of the $a$ locus (Banuett and Herskowitz 1989) and might occur in response to plant signals (Banuett 1992). It is possible that $f u z 7^{-}$mutants fail to develop germination competence owing, for example, to defective fungal differentiation. Another possibility is that fuz7 is part of another pathway that responds to environmental cues (such as osmolarity) that govern germination itself.

\section{fuz7 participates in at least two pathways}

We summarize our view of fuz7 in the life cycle of $U$. maydis in Figure 8. Our findings indicate that Fuz7, a MEK/MAPKK homolog, participates in at least two different pathways that respond to different signals: In one pathway, the signal is provided by the pheromones encoded by the $a$ locus; in the other pathway, the signal is hypothesized to be of plant origin. It is thought that activation of $f u z 7$ by pheromones leads to activation of a putative MAP kinase (shown as MAPKl in Fig. 8), which, in turn, leads to activation of conjugation tube formation and mating. Once the fusion product is formed, this pathway is also activated by the pheromones in an autocrine response to maintain the filamentous state. Activation of $f u z 7$ by the putative plant signal may stimulate a different MAP kinase (MAPK2), which, in turn, is responsible for tumor induction and perhaps for acquisition of germination competence by the teliospores. The budding yeast MEK/MAPKK homolog Ste7 can also be activated by two different signals, the mating pheromones and another signal yet to be determined (Fig. 8). The studies with a MAPKK/MEK homolog of $U$. maydis demonstrate once again the ubiquity and versatility of the MAP kinase pathway.

\section{Materials and methods}

U. maydis strains are described in Table 1.

\section{Plant inoculations and growth conditions}

Corn variety B164 was used in all studies. Plants were grown in a Conviron chamber with a 14 -hr light $/ 10$-hr dark cycle at $28^{\circ} \mathrm{C}$ day temperature $/ 20^{\circ} \mathrm{C}$ night temperature.

Saturated cultures $\left(10^{8} \mathrm{cells} / \mathrm{ml}\right)$ of haploid strains were mixed in a $1: 1$ ratio, and $0.2 \mathrm{ml}$ of this mixture or $0.2 \mathrm{ml}$ of a saturated culture of diploid strains was inoculated into 3- to 5-day-old seedlings using a hypodermic syringe. Inoculations were repeated five to six times each day on three independent days. Symptom development was followed for 1 month.

\section{Media and growth conditions for Ustilago strains}

YEPS (Tsukuda et al. 1988) was used to grow cultures for most manipulations. UMC broth $(U$. maydis complete; Holliday 1974) was used for growing strains for the fuzz assay (Banuett and Herskowitz 1989) or for plant inoculations. The fuzz reaction was carried out on UMC agar containing $1 \%$ charcoal /Holliday 1974). Strains to be tested were cross-streaked against standard tester strains $[a 1 b 1$ (FB1), $a 2$ b2 (FB2), $a 2 b 1$ (FB6a), $a 1$ $b 2$ [FB6b) on this medium. Plates were sealed with parafilm and incubated at room temperature. Fuz ${ }^{+}$strains exhibit an intense reaction after $24 \mathrm{hr}$, whereas Fuz strains exhibit a reduced reaction or no reacton even after $48-72 \mathrm{hr}$ of incubation (see Banuett 1991). 
Figure 8. Fuz7 is involved in two signaling pathways. (Left) Summary of the two different pathways in which the MEK/MAPKK homolog Fuz7 is proposed to participate. (Right) Summary of the two pathways in which the MEK/MAPKK homolog STE7 is involved in the life cycle of $S$. cerevisiae. Additional details are described in the text.
U.maydis

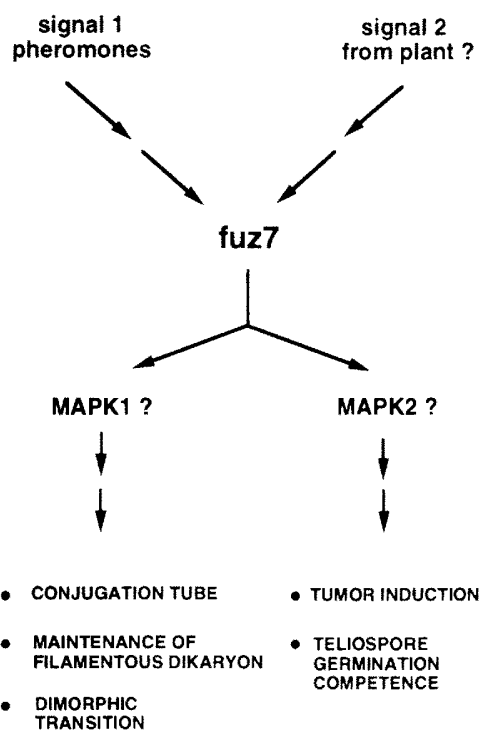

\section{S. cerevisiae}

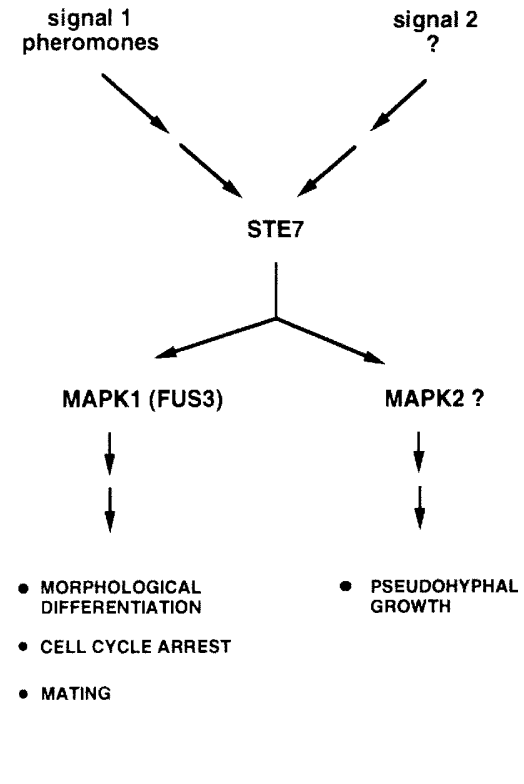

Minimal medium (MM) (Holliday 1974), was supplemented with $1 \mathrm{M}$ sorbitol for growth of protoplasts.

Conjugation tube assay media and conditions are described by Banuett and Herskowitz (1994).

\section{Genetic methods}

UV mutagenesis to generate auxotrophs Strains FB6a and FB6b (Table 1) were mutagenized by irradiation with UV light to $5 \%$ survival (see Banuett 1991) and plated on YEPS medium; colonies were then replica-plated onto MM plates. Growth requirements of mutants were determined on supplemented MM.

DNA-mediated transformation of $\mathrm{U}$. maydis Strains were grown in YEPS broth and transformed according to Wang et al. (1988). Transformants were selected on YEPS hygromycin B $(400 \mu \mathrm{g} / \mathrm{ml})$ plates with an overlay of YEPS $3 \%$ agar without drug. For gene replacement, the DNA fragment to be transformed was first gel-fractionated and purified using a QIAEX kit (Qiagen). Transformants were purified on YEPS agar containing $200 \mu \mathrm{g} / \mathrm{ml}$ of hygromycin $B$.

Teliospore analysis Teliospores were recovered from the tumors (see Banuett 1991) and germinated on YEPS agar slabs. For random spore analysis, minicolonies containing the meiotic products were resupended in water and plated on YEPS agar to obtain single colonies. For determination of germination frequency, large numbers of teliospores were spread on YEPS slabs and scored for promycelium formation. In some cases, tetrad analysis was performed as follows: Teliospores were allowed to germinate and produce a promycelium (a short filament extruded from the spore). Meiosis occurs within the promycelium to yield primary meiotic products (a four-celled septate filament), each of which is capable of producing long chains of progeny haploid cells (sporidia) by mitotic divisions (O'Donnell and McLaughlin 1984). Sporidia were removed by micromanipulation, grown into colonies, and tested as appropriate.
Protoplast fusion conditions and selection of diploids Diploid strains FBD-13, FBD-14, FBD-15, and FBD7-7H were constructed by protoplast fusion (Table 1). Parental strains were grown in YEPS to $5 \times 10^{7}$ cells $/ \mathrm{ml}$ and mixed as described in Table 1. Protoplasts were prepared from each mixture, treated with PEG as for transformation, and plated on MM containing sorbitol to select prototrophs.

Homozygosity of the fuz 7 mutation and heterozygosity at $b$ were corroborated by Southern hybridization (data not shown).

\section{DNA manipulations}

DNA isolation, plasmid manipulations, and Southern hybridization Standard procedures for plasmid manipulations were followed (Ausubel et al. 1988; Maniatis et al. 1989). U. maydis DNA was isolated from $50-\mathrm{ml}$ cultures grown in YEPS broth to $2 \times 10^{7}$ to $4 \times 10^{7}$ cells $/ \mathrm{ml}$. Cells were washed once with water and resuspended in sodium citrate buffer (Wang et al. 1988). Protoplasts were prepared using Novozyme234 (Fungal Genetics Stock Center) and lysed with SDS/NaOH. The lysate was extracted several times with phenol-chloroform and ethanol precipitated. The DNA was resuspended in Tris-EDTA $110 \mathrm{~mm}$ Tris, $1 \mathrm{~mm}$ EDTA), and treated with RNase, and the phenol extraction and ethanol precipitation steps were repeated. The DNA thus prepared was suitable for restriction endonuclease digestion and for PCR amplification.

Southern hybridization was performed as described in Maniatis et al. (1989), with minor modifications. To determine the structure at the fuz 7 locus, genomic DNA was treated with restriction endonucleases $B g l I I$ or $E c o$ RI, fractionated on an agarose gel, and transferred to a nitrocellulose filter (Schleicher \& Schuell). The filters were probed with an EcoRI fragment (see Fig. 3), radioactively labeled with $\left[\alpha^{-32} \mathrm{p}\right] \mathrm{dCTP}$ according to the instructions provided in the Pharmacia random labeling kit. To determine heterozygosity at $b$ of the diploid strains, genomic DNA was treated with both BgIII and SalI, transferred as above, and filters were probed with a $b 2$ BgIII-SalI fragment labeled as 
above. Under these conditions, two different fragments are detected in a $b 1 / b 2$ strain: a $1.5-\mathrm{kb}$ fragment corresponding to $b 1$ and a $0.9-\mathrm{kb}$ fragment corresponding to $b 2$.

DNA sequencing and sequence analysis Double-strand sequencing was performed using the Sequenase 2.0 sequencing kit (U.S. Biochemical) with universal primers or custom synthesized primers. The templates (pUC-derived plasmids or a pBR322-derived cosmid/ were denatured as described in Ausubel et al. (1988).

Alignment of the Fuz7 protein sequence with other members of the MEK/MAPKK family was first performed by eye and refined by use of the GeneWorks program (IntelliGenetics).

\section{PCR amplification}

Conditions Amplification of $f u z 7$ from genomic and plasmid DNA with degenerate primers was performed in a Perkin-Elmer DNA thermal cycler with 40 cycles of amplification using AmpliTaq (Perkin-Elmer Cetus). Each cycle consisted of 1-min denaturation at $94^{\circ} \mathrm{C}, 1-\mathrm{min}$ annealing of primers at $42^{\circ} \mathrm{C}$ and 1 -min extension at $72^{\circ} \mathrm{C}$. A 15 -min extension at $72^{\circ} \mathrm{C}$ was performed after completion of the 40 cycles. One to ten nanograms of DNA was used for analysis of the cosmid library; 10-50 ng was used for amplification of genomic DNA. Degenerate primers AN9 and AN11 (Fig. 1) were used for each step in the sibselection procedure. As controls, pSTE7 and pbyr1 (Neiman et al. 1993) were amplified using the same conditions.

Oligonucleotides AN9 and AN11 were synthesized by Operon Biotechnologies. AN9 is $5^{\prime}-\mathrm{CC}[\mathrm{A}, \mathrm{C}, \mathrm{G}, \mathrm{T}] \mathrm{TA}[\mathrm{C}, \mathrm{T}]$ AT[A,C,T] GT[A,C,G,T] GA[C,T] TT[C,T, TT[C,T] TA[C,T] GG[A,C,G,T] GC and AN11 is $5^{\prime}-\mathrm{GG}[\mathrm{A}, \mathrm{C}, \mathrm{G}, \mathrm{T}][\mathrm{G}, \mathrm{C}][\mathrm{A}, \mathrm{T}] \mathrm{C}$ AT[G,A] TA[A,C,G,T] GT[A,C,G,T] [G,C][A,T][A,C,G,T] GT[A,C,G,T] CC. Primers FB133 and FB134 and all primers for DNA sequencing were synthesized using a MilliGen/Biosearch Cyclone Plus DNA synthesizer. FB133 is $5^{\prime}$-CGG AGA TCT TGG ATC CCT GAG CCA and FB134 is 5'-CGG CAA GGA TCC GCC AGT CTT.

Sib selection of library by PCR Sib selection of a cosmid library using PCR was performed as follows using the conditions described above: Twenty pools of a cosmid bank containing a total of 3000 clones were screened by PCR with primers AN9 and AN11 (Fig. 1). The band corresponding to byr1 was amplified in six of the pools, of which two ( 3 and 11) were studied further. These pools were further divided into smaller subpools that were subjected to the same PCR conditions. This procedure identified cosmid p11F23-8 containing a $40-\mathrm{kb}$ insert that presumably carries the $S T E 7 /$ byr 1 homolog. Upon digestion of p11F23-8 with restriction endonuclease BglII, the seven fragments produced were subcloned into the BamHI site of pUCl8 and transformed into Escherichia coli strain DH5 $\alpha$ (Bethesda Research Laboratories). Transformant colonies were screened by restriction enzyme analysis and then by PCR. Plasmid pBB2, containing a $1.8-\mathrm{kb}$ insert, was thus identified.

\section{Delection of fuz7 by one-step gene replacement}

The entire fuz 7 gene was amplified from cosmid p11F23-8 with primers FB133 and FB134, which contain BamHI sites to facilitate subcloning. A $2.3-\mathrm{kb}$ band was amplified with 25 cycles consisting of 1 -min denaturation at $94^{\circ} \mathrm{C}, 2$-min annealing/extension at $68^{\circ} \mathrm{C}$, and $15-\mathrm{min}$ extension at $72^{\circ} \mathrm{C}$ at the end of the 25 cycles. The amplified DNA was treated with restriction endonuclease BamHI and ligated to the BamHI site of plasmid p18F, a pUC18 derivative with an Xbal-HindIII deletion, to generate plasmid pl8F- $f 7$. The insert in this plasmid was se- quenced to confirm that no errors had been introduced during amplification. Plasmid p18F-f7 was then treated with restriction endonuclease HincII, which cuts only within the fuz7 ORF, thus deleting 1092 nucleotides within it (Figs. 1 and 3). A 3.0-kb Pvull fragment from plasmid pHL1 (Wang et al. 1988) containing the hygromycin B gene was ligated to the Hincll-deleted plasmid to generate plasmid $\mathrm{p} 18 \mathrm{~F}-\mathrm{f} 7:: \mathrm{H}$. This plasmid was treated with restriction endonuclease $B a m H I$, and the BamHI fragment containing the fuz $7::$ hygB mutation was gel-purified and used for transformation of $U$. maydis strains FB1, FB2, FB37-7, and FB90-8 (Table 1).

\section{Light microscopy}

Cells were routinely observed with Nomarski interference optics using a Zeiss axioscope equipped with an MC100 or Polaroid camera.

\section{Acknowledgments}

We are very thankful to Aaron Neiman for providing primers AN9 and AN11. We also thank James DeVay for the kind gift of B164 seeds and Marlis Dahl and Regine Kahmann for the $U$. maydis cosmid library. This work was supported by research grant AI 18738 from the National Institutes of Health and by the Albert Bowers Fund.

The publication costs of this article were defrayed in part by payment of page charges. This article must therefore be hereby marked "advertisement" in accordance with 18 USC section 1734 solely to indicate this fact.

\section{References}

Achstetter, T. 1989. Regulation of $\alpha$-factor production in Saccharomyces cerevisiae: $\alpha$-Factor pheromone-induced expression of the MFo1 and STE13 genes. Mol. Cell. Biol. 9: 45074514.

Ausubel, F., R. Brent, R.E. Kingston, D.D. Moore, J.G. Seidman, J.A. Smith, and K. Struhl. 1988. Current protocols in molecular biology. Greene Publishing Associates/Wiley-Interscience, New York.

Ballance, D.J. 1991. Transformation systems for filamentous fungi and an overview of fungal gene structure. In Molecular industrial mycology. Systems and applications for filamentous fungi (ed. S.A. Leong and R.M. Berka), vol. 8, pp. 1-29. Marcel Dekker, New York.

Banuett, F. 1991. Genes required for filamentous growth and tumor induction by the corn smut fungus Ustilago maydis. Proc. Natl. Acad. Sci. 88: 3922-3926.

- 1992. Ustilago maydis, the delightful blight. Trends Genet. 8: 174-180.

Banuett, F. and I. Herskowitz. 1989. Different $a$ alleles of Ustilago maydis are necessary for maintenance of filamentous growth but not for meiosis. Proc. Natl. Acad. Sci. 86: 58785882 .

-1994. Morphological transitions in the life cycle of Ustilago maydis and their genetic control by the $a$ and $b$ loci. Exp. Mycol. (in press).

Boguslawski, G. and J.O. Polazzi. 1987. Complete nucleotide sequence of a gene conferring polymyxin B resistance on yeast: Similarity of the predicted polypeptide to protein kinases. Proc. Natl. Acad. Sci. 84: 5848-5852.

Bölker, M., M. Urban, and R. Kahmann. 1992. The a mating type locus of $U$. maydis specifies cell signalling components. Cell 68: $441-450$. 
Brewster, J.L., T. de Valoir, N.D. Dwyer, E. Winter, and M.C. Gustin. 1993. An osmosensing signal transduction pathway in yeast. Science 259: 1760-1763.

Casselton, L. 1978. Dikaryon formation in higher Basidiomycetes. In The filamentous fungi led. J.E. Smith and D.R. Berry), vol. 3, pp. 275-297. Arnold Press, London, UK

Crews, C.M. and R.L. Erikson. 1993. Extracellular signals and reversible protein phosphorylation: What to MEK of it all. Cell 74: 215-217.

Crews, C.M., A. Alessandrini, and R.L. Erikson. 1992. The primary structure of $M E K$, a protein kinase that phosphorylates the ERK gene product. Science 258: 478-480.

Dénarié, J. and J. Cullimore. 1993. Lipo-oligosaccharide nodulation factors: New class of signalling molecules mediating recognition and morphogenesis. Cell 74: 951-954.

Duntze, W., V.L. MacKay, and T.R. Manney. 1970. Saccharomyces cerevisiae: A diffusible sex factor. Science 168: 14721474.

Fotheringham, S. and W.K. Holloman. 1989. Cloning and disruption of Ustilago maydis genes. Mol. Cell. Biol. 9: 40524055 .

Hanks, S.K., A.M. Quinn, and T. Hunter. 1988. The protein kinase family: Conserved features and deduced phylogeny of the catalytic domains. Science 241: 42-52

Hartwell, L. 1980. Mutants of Saccharomyces cerevisiae unresponsive to cell division control by polypeptide mating hormone. J. Cell Biol. 85: 811-822.

Holliday, R. 1974. Ustilago maydis. In Handbook of genetics (ed. R.C. King), vol. 1, pp. 575-595. Plenum, New York.

Irie, K., M. Takase, K.S. Lee, D.E. Levin, H. Araki, K. Matsumoto, and Y. Oshima. 1993. $M K K 1$ and $M K K 2$, which encode Saccharomyces cerevisiae mitogen-activated protein kinasekinase homologs, function in the pathway mediated by protein kinase C. Mol. Cell. Biol. 13: 3076-3083.

Kosako, H., E. Nishida, and Y. Gotoh. 1993. cDNA cloning of MAP kinase kinase reveals kinase cascade pathways in yeasts to vertebrates. $E M B O$ J. 12: 787-794

Lee, K.S., K. Irie, Y. Gotoh, Y. Watanabe, H. Araki, E. Nishida, K. Matsumoto, and D. Levin. 1993. A yeast mitogen-activated protein kinase homolog (Mpklp) mediates signalling by protein kinase C. Mol. Cell. Biol. 13: 3067-3075.

Liu, H., C.A. Styles, and G.F. Fink. 1993. The mating kinase cascade of Saccharomyces cerevisiae is required in diploids for filamentous growth. Science 262: 1741-1744.

Long, S.R. and B.J. Staskawicz. 1993. Prokaryotic plant parasites. Cell 73: 921-935.

Maniatis, T., E.F. Fritsch, and J. Sambrook. 1989. Molecular cloning: A laboratory manual. Cold Spring Harbor Laboratory Press, Cold Spring Harbor, New York.

Marsh, L., A.M. Neiman, and I. Herskowitz. 1991. Signal transduction during pheromone response in yeast. Annu. Rev. Cell Biol. 7: 699-728.

Nadin-Davis, S.A. and A. Nasim. 1988. A gene which encodes a predicted protein kinase can restore some functions of the ras gene in fission yeast. EMBO J. 7: 985-993.

Neiman, A.M. 1993. Conservation and reiteration of a kinase cascade. Trends Genet. 9: 390-394.

Neiman, A.M., B.J. Stevenson, H.-P. Xu, G.F. Sprague, I. Herskowitz, M. Wigler, and S. Marcus. 1993. Functional homology of protein kinases required for sexual differentiation in Schizosaccharomyces pombe and Saccharomyces cerevisiae suggests a conserved signal transduction module in eukaryotic organisms. Mol. Biol. Cell 4: 107-120.

Nishida, E. and Y. Gotoh. 1993. The MAP kinase cascade is essential for diverse signal transduction pathways. Trends Biochem. 18: 128-131.
Novotny, C.P., M.M. Stankis, C.A. Specht, H. Yang, and R.C.

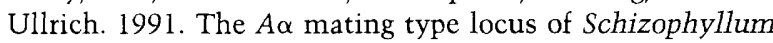
commune. In More gene manipulations in fungi (ed. J.W. Bennett and L.L. Lasure), pp. 234-257. Academic Press, New York/London, UK.

O'Donnell, K.L. and D.J. McLaughlin. 1984. Ultrastructure of meiosis in Ustilago maydis. Mycologia 76: 468-485.

Rowell, J.B. 1955. Functional role of compatibility factors and an in vitro test for sexual compatibility with haploid lines of Ustilago zeae. Phytopathology 45: 370-374.

Rowell, J.B. and J.E. DeVay. 1954. Genetics of Ustilago zeae in relation to basic problems of its pathogenicity. Phytopathology 44: 356-362.

Strazdis, J.R. and V.L. MacKay. 1983. Induction of yeast mating pheromone a-factor by $\alpha$ cells. Nature 305: $543-545$.

Tanaka, K., J. Davey, Y. Imai, and M. Yamamoto. 1993. Schizosaccharomyces pombe map $3^{+}$encodes the putative M-factor receptor. Mol. Cell. Biol. 13: 80-88.

Teague, M.A., D.T. Chaleff, and B. Errede. 1986. Nucleotide sequence of the yeast regulatory gene $S T E 7$ predicts a protein homologous to protein kinases. Proc. Natl. Acad. Sci. 83: $7371-7375$.

Trueheart, I. and I. Herskowitz. 1992. The $a$ locus governs cytoduction in Ustilago maydis. I. Bacteriol. 174: 7831-7833.

Tsuda, L., Y.H. Inoue, M.-A. Yoo, M. Mizuno, M. Hata, Y.-M. Lim, T. Adachi-Yamada, H. Ryo, Y. Masamune, and Y. Nishida. 1993. A protein kinase similar to MAP kinase activator acts downstream of the Raf kinase in Drosophila. Cell 72: 407-414.

Tsukuda, T., S. Carleton, S. Fotheringham, and W.K. Holloman. 1988. Isolation and characterization of an autonomously replicating sequence from Ustilago maydis. Mol. Cell. Biol. 8: 3703-3709.

Wang, J., D.W. Holden, and S.A. Leong. 1988. Gene transfer system for the phytopathogenic fungus Ustilago maydis. Proc. Natl. Acad. Sci. 85: 865-869.

Warbrick, E. and P.A. Fantes. 1991. The wisl protein kinase is a dosage-dependent regulator of mitosis in Schizosaccharomyces pombe. EMBO J. 10: 4291-4299. 


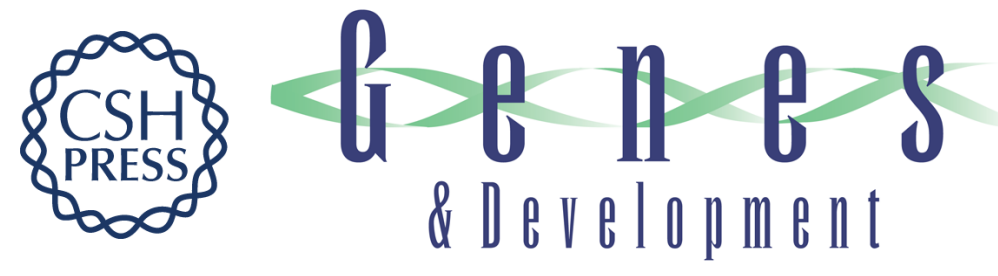

\section{Identification of fuz7, a Ustilago maydis MEK/MAPKK homolog required for a-locus-dependent and-independent steps in the fungal life cycle.}

F Banuett and I Herskowitz

Genes Dev. 1994, 8:

Access the most recent version at doi:10.1101/gad.8.12.1367

References This article cites 35 articles, 19 of which can be accessed free at:

http://genesdev.cshlp.org/content/8/12/1367.full.html\#ref-list-1

License

Email Alerting Service

Receive free email alerts when new articles cite this article - sign up in the box at the top right corner of the article or click here.

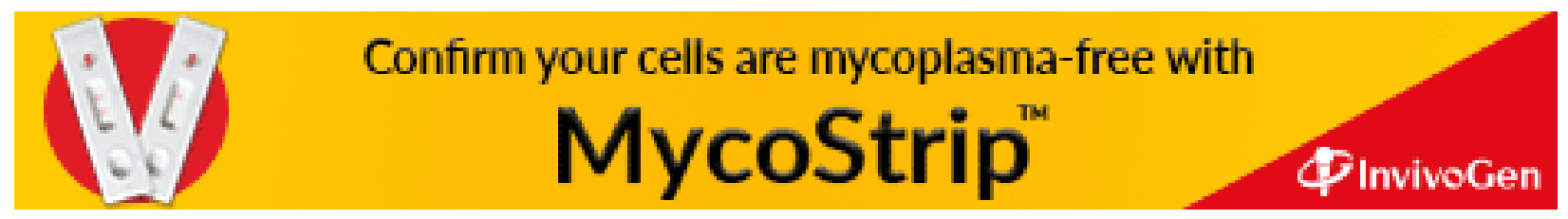

